\title{
Femtosecond laser micromachining of compound parabolic concentrator fiber tipped glucose sensors
}

\author{
Hafeez Ul Hassan \\ Amédée Lacraz \\ Kyriacos Kalli \\ Ole Bang
}




\title{
Femtosecond laser micromachining of compound parabolic concentrator fiber tipped glucose sensors
}

\author{
Hafeez UI Hassan, ${ }^{a, \star}$ Amédée Lacraz, ${ }^{b}$ Kyriacos Kalli, ${ }^{b}$ and Ole Bang ${ }^{a}$

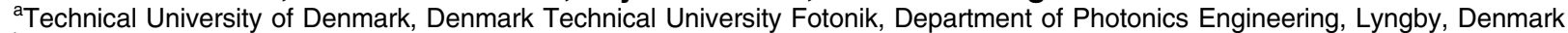 \\ ${ }^{b}$ Cyprus University of Technology, Nanophotonics Research Group, Department of Electrical Engineering, Computer Engineering and Informatics, \\ Limassol, Cyprus
}

\begin{abstract}
We report on highly accurate femtosecond (fs) laser micromachining of a compound parabolic concentrator (CPC) fiber tip on a polymer optical fiber (POF). The accuracy is reflected in an unprecedented correspondence between the numerically predicted and experimentally found improvement in fluorescence pickup efficiency of a Förster resonance energy transfer-based POF glucose sensor. A Zemax model of the CPC-tipped sensor predicts an optimal improvement of a factor of 3.96 compared to the sensor with a plane-cut fiber tip. The fs laser micromachined CPC tip showed an increase of a factor of 3.5 , which is only $11.6 \%$ from the predicted value. Earlier state-of-the-art fabrication of the CPC-shaped tip by fiber tapering was of so poor quality that the actual improvement was $43 \%$ lower than the predicted improvement of the ideal CPC shape. $\odot$ The Authors. Published by SPIE under a Creative Commons Attribution 3.0 Unported License. Distribution or reproduction of this work in whole or in part requires full attribution of the original publication, including its DOI. [DOI: 10.1117/1.JBO.22.3.037003]
\end{abstract}

Keywords: fiber optics sensors; polymers; blood or tissue constituent monitoring; nonimaging optics.

Paper 160876R received Dec. 23, 2016; accepted for publication Feb. 21, 2017; published online Mar. 14, 2017.

\section{Introduction}

Fluorescence-based polymer optical fiber (POF) glucose sensors for continuous glucose monitoring have significant potential for optimum control of diabetes. ${ }^{1-3}$ One limitation of fluorescencebased sensors is that the detected fluorescence signal is often weak, which results in low signal-to-noise ratio, ${ }^{4}$ and significantly compromises the sensor performance. This problem can be overcome in fiber-optical fluorescence sensors by shaping the fiber tip to increase the numerical aperture (NA) of the fiber thereby collecting more of the fluorescence signal, as demonstrated by Gao et al. ${ }^{5}$ by linear and step-wise tapering of silica fibers. In our previous work on POF-based glucose sensors, ${ }^{6}$ we have shaped the POF tip as a compound parabolic concentrator (CPC), which is a well-known profile that provides optimal light pickup efficiency, ${ }^{7}$ as demonstrated in solar energy systems for thermal and photovoltaic applications ${ }^{8}$ and for coupling light emitting diode (LED) light into fibers. ${ }^{9}$

In our original work, CPC tips were fabricated by parabolically tapering of the POF using the heat-and-pull method and demonstrated to increase the NA and fluorescence light pickup efficiency of the fiber. However, the CPC fiber tips fabricated by the heat-and-pull method were not geometrically close to the ideal CPC shape, as shown in Fig. 1, but had imperfections, such as an increase in the local diameter at the start of the CPC tip. Such imperfections led to a $43 \%$ reduction in the maximum fluorescence that could have been achieved by the ideal CPC shape, as predicted by Zemax modeling. ${ }^{6}$ To take full advantage of the CPC to achieve maximum increase in the fluorescence pickup efficiency, an alternative approach is needed to allow better control and make fiber tips with a shape closer to the ideal CPC shape. One such powerful technique to shape fiber tips is

*Address all correspondence to: Hafeez UI Hassan, E-mail: hafha@fotonik. dtu.dk femtosecond (fs) laser micromachining. fs lasers have been used for micromachining ${ }^{10}$ and for fabricating fiber Bragg gratings, ${ }^{11}$ optical waveguides, ${ }^{12,13}$ and several photonics devices ${ }^{14-16}$ in transparent materials, such as glass. Several groups have also used fs lasers for micromachining in polymers, ${ }^{17,18}$ to write POF FBGs ${ }^{19,20}$ and fabricate photonic devices. ${ }^{21,22}$ fs lasers have the ability to precisely structure different materials in three dimensions, which make it a powerful technique to micromachine and shape the optical fiber tips. ${ }^{23-25}$

In this paper, we report for the first time the fs laser micromachining of a CPC shaped POF tip with a shape that is close to the ideal CPC, as shown in Fig. 1. We further demonstrate that the fs laser machined high-quality CPC tip gives an improvement of the pickup efficiency of a factor of 3.5 , as compared to the corresponding plane-cut fiber tip. This is only an $11.6 \%$ deviation from the increase a perfect CPC shape of that length should give. The previous state of the art in pickup efficiency improvement with a CPC tip was $43 \%$ lower than the predicted optimum. $^{6}$

\section{Femtosecond-Laser Micromachining of Compound Parabolic Concentrator}

The CPC in two-dimensional geometry is formed by two identical and oppositely aligned parabolas truncated at their focal point, as shown in Fig. 2(a). The CPC profile is traced by the coordinates $y$ and $z$, as defined in Ref. 9 and given by

$$
\begin{aligned}
& y=\frac{2 a_{1}\left(1+\sin \theta_{i}\right) \sin \left(\varphi-\theta_{i}\right)}{1-\cos \varphi}-a_{1} \\
& z=\frac{2 a_{1}\left(1+\sin \theta_{i}\right) \cos \left(\varphi-\theta_{i}\right)}{1-\cos \varphi}
\end{aligned}
$$

where $a_{1}$ and $a_{2}$ are the radii of the input and output apertures, $\theta_{i}$ is the acceptance angle of the $\mathrm{CPC}$, and $\varphi$ is an angle that varies 
(a) Ideal

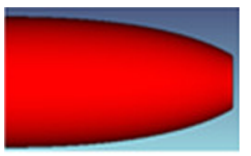

(b) Heat and pull

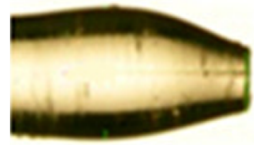

(c) Micromachined

Fig. 1 (a) Ideal CPC shape. (b) Nonoptimal CPC fabricated by the heat-and-pull method. (c) fs laser micromachined CPC close to the ideal shape.

from $2 \theta_{i}$ to $90 \mathrm{deg}+\theta_{i}$. The geometrical parameters of the CPC are related through the following equation:

$a_{1}=a_{2} / \sin \theta_{i} ; \quad L=\left(a_{1}+a_{2}\right) / \tan \theta_{i}$,

where $L$ denotes the CPC length. A CPC conserves the entendue, $a_{1} \mathrm{NA}_{1}=a_{2} \mathrm{NA}_{2},{ }^{7}$ which means that the NA at the output is increased due to the reduced output aperture. Therefore, the $\mathrm{CPC}$ tip can increase the fluorescence pickup efficiency in POFbased glucose sensors, as reported in our previous work ${ }^{6}$ for a 35-mm long sensor.

The fiber used to manufacture the CPC tip is the Super ESKA fiber with a polymethyl methacrylate (PMMA) core and polyvinylidene difluoride (PVDF) cladding. The core diameter is $240 \mu \mathrm{m}$ and the outer diameter is $250 \mu \mathrm{m}$. The refractive index of the core and cladding is $n_{\text {core }}=1.49$ and $n_{\text {clad }}=1.40$, respectively, which results in an NA of 0.5. It is known from Ref. 9 that all of the light collected by the CPC can be coupled perfectly to the straight part of the fiber only if the acceptance angle of the $\operatorname{CPC}\left(\theta_{i}\right)$ is within the acceptance angle of the straight fiber $\left(\theta_{\max }\right)$, i.e., if $\theta_{i} \leq \theta_{\max }=90 \mathrm{deg}-\theta_{c}$, where $\theta_{c}=$ $\sin ^{-1}\left(n_{\text {clad }} / n_{\text {core }}\right)$ is the critical angle for total internal reflection at the core-cladding interface. The maximum CPC acceptance angle is then $\theta_{i}=\theta_{\text {max }}=\cos ^{-1}\left(n_{\text {clad }} / n_{\text {core }}\right)$. For this $\theta_{i}$ and the input aperture $2 a_{1}=240 \mu \mathrm{m}$, Eq. (2) gives the CPC output aperture $2 a_{2}=82 \mu \mathrm{m}$ and the CPC length $L=445 \mu \mathrm{m}$. These CPC parameters were used in our previously defined Zemax model of the sensor, ${ }^{6}$ which showed that the ideal CPC shape of the tip would increase the detected fluorescence by a factor of 3.96 compared to the plane-cut fiber tip.

Before micromachining this fiber tip, the fiber PVDF cladding was removed using triethyl phosphate amide (solvent for
PVDF) ${ }^{26}$ for a length of $450 \mu \mathrm{m}$, which was the closest we could get to the ideal $445 \mu \mathrm{m}$. The fiber was then machined using the fs-laser setup shown in Fig. 2(b). The setup consists of an fs-laser system (HighQ laser femtoREGEN) operating at $517 \mathrm{~nm}$, with a $250-\mathrm{fs}$ pulse duration, 380-nJ pulse energy, and $100-\mathrm{kHz}$ repetition rate. The fiber was mounted as shown in Fig. 2 on an air bearing translation system (Aerotech) for a nanometer accurate two-axis motion in the $x$ - and $y$-directions and the laser beam was focused from above using a long working distance $50 \times$ objective (Mitutoyo). The CPC shape is then carved out from the top-down in 63 steps of $7 \mu \mathrm{m}$ in the direction of the fiber axis, as shown in Fig. 2(c), with the first tip diameter set at $82 \mu \mathrm{m}$. For each step, the carving was undertaken in circles in a number of rounds given by the laser beam width $(2 \mu \mathrm{m})$ and the desired tip diameter, increasing the radius of the circle by $1 \mu \mathrm{m}$ each round. The increase in tip diameter was made such as to follow Eq. (2).

\section{Glucose Sensor}

The fs-laser micromachined CPC POF tips were tested in the POF-based glucose sensor, whose sensor principle is detailed in Fig. 3. The glucose sensor, which is the same as the one also used in our previous work, ${ }^{6}$ uses Förster resonance energy transfer $(\text { FRET) })^{27}$ as the sensing mechanism. FRET is a radiation less energy transfer between an excited donor fluorophore and a proximal ground state acceptor (a fluorophore or a dye) through a long range dipole-dipole interaction and can be used to measure intermolecular distances in the range of angstroms (10 to $100 \AA){ }^{27}$

The assay chemistry of the sensor is filled in a compartment, which is formed by bonding a glucose permeable membrane onto the fiber. Glucose molecules enter into the assay through the permeable membrane, when the sensor is place in the glucose solution. The assay chemistry consists of a glucose binding protein labeled with a fluorophore (Alexa fluor 594: $\lambda_{\text {excitation }}=$ $590 \mathrm{~nm}, \lambda_{\text {emission }}=618 \mathrm{~nm}$ ) and a glucose analog (green chain) labeled with crystal violet dye (blue dots). Together they make an FRET pair resulting in the quenching of the fluorophore, thus reducing the emitted fluorescence intensity. When the glucose molecules enter the chemistry, they compete with the glucose analog molecules to attach to the protein. This competition results in breaking the FRET pair and changing their quantity (a)

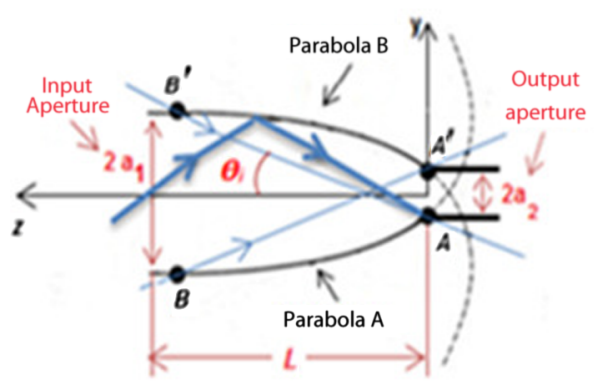

(b)

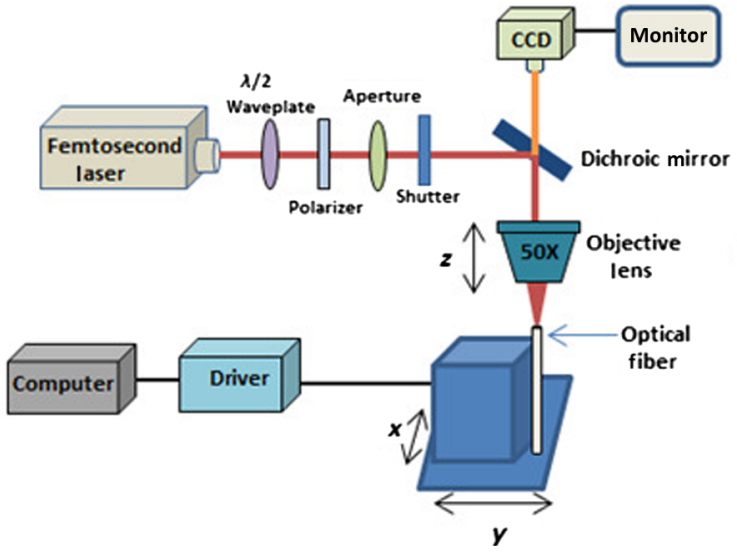

(c)

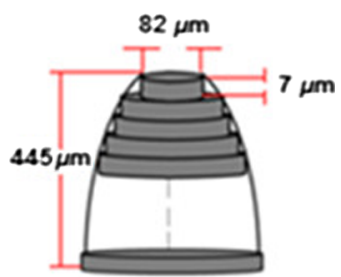

Fig. 2 (a) Geometry of a CPC. (b) fs laser setup with stages to micromachine the fiber tip. (c) Illustration of carving process. 
(a)

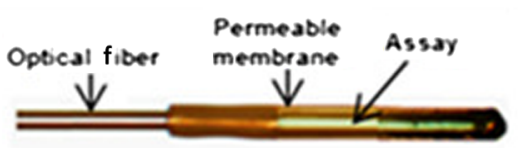

(c)

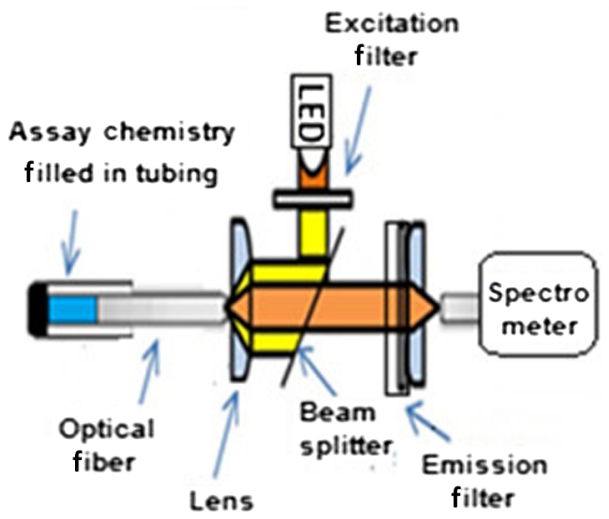

(b)
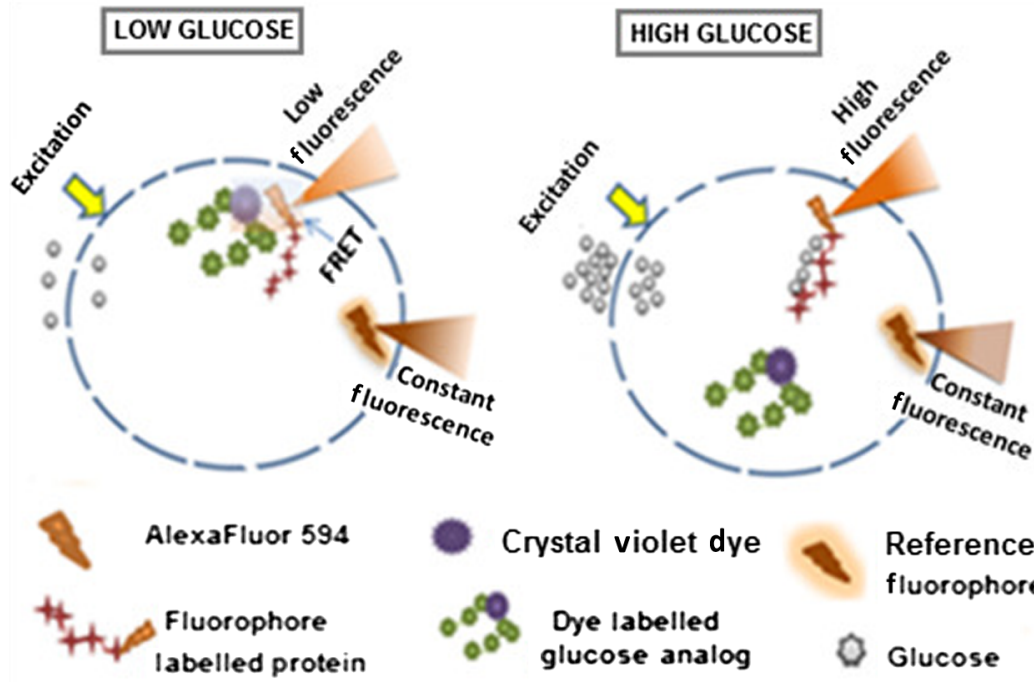

Crystal violet dye

Reference fluorophore

Dye labelled glucose analog

Glucose

Fig. 3 (a) Real glucose sensor. (b) Illustration of the assay chemistry and FRET sensing principle resulting in low and high intensity. (c) Optical setup for sensor characterization.

in the chemistry. When equilibrium is reached, the reduced number of FRET pairs can be detected as an increase in the overall intensity of the fluorescence collected by the fiber. Therefore, the glucose concentration can be correlated to the detected fluorescence and used to measure the glucose concentration.

The sensor chemistry also contains a reference fluorophore (Alexa fluor $700: \lambda_{\text {excitation }}=633-647 \mathrm{~nm}, \lambda_{\text {emission }}=723 \mathrm{~nm}$ ), which is unaffected by the glucose concentration. This is to eliminate any unwanted fluctuations in the detected fluorescence intensity caused either by the fluctuations in the light source used to excite the assay or in the coupling between the assay and the light source. The ratio between the assay and the reference fluorescence determines the absolute glucose concentration.

The sensors are characterized by an epifluorescence setup and a fiber-optical spectrometer (USB2000+, Ocean Optics, Florida), as shown in Fig. 3(c). In the setup, the light from the LED source (HLMP-EL30-MQ000), with central wavelength $590 \mathrm{~nm}$, passes through a 55-nm excitation filter centered at $560 \mathrm{~nm}$ and then through a beam splitter to finally be coupled to the fiber by a lens, in order to excite the assay chemistry. The resulting fluorescence from the assay is collected by the fiber and passes through the beam splitter and a long-pass emission filter with 610-nm cut-off wavelength to finally reach the spectrometer.

\section{Results and Discussions}

The final fs laser machined CPC shape is shown in Fig. 4(a). To make the surface of the CPC smoother, it is dipped into dibromomethane, a solvent for PMMA, ${ }^{28}$ for $20 \mathrm{~s}$ and then cleaned with distilled water.

The fluorescence coupling efficiency of the CPC-tipped and plane-cut fiber sensors were both characterized in a dummy sensor configuration, using a miniature cuvette made of a nonfluorescing transparent $250 \mu \mathrm{m}$ inner diameter Tygon tube sealed in one end. The glucose chemistry is filled into the tube and the $35-\mathrm{mm}$ long fiber is inserted into the tube [see Fig. 3(c)], (a)

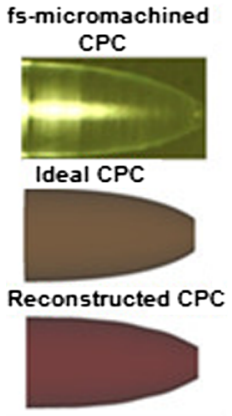

(b)

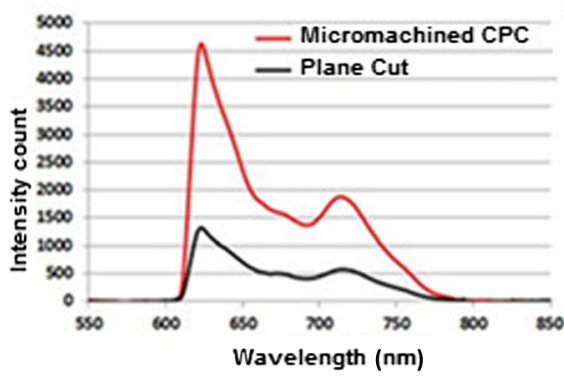

Fig. 4 (a) From top to bottom: fs-laser micromachined CPC, ideal $\mathrm{CPC}$, and reconstructed CPC shape in Zemax. (b) Average fluorescence spectra from plane-cut and micromachined CPC tips, each averaged over three measurements.

and characterized using the optical setup described in Sec. 3. To avoid any effect of misalignment and coupling variations from the setup, three measurements are taken for each sensor, each using a new dummy sensor.

The detected average spectra from the CPC-tipped and planecut fiber sensors are shown in Fig. 4(b); each has been averaged over three measurements. We define the increment factor as the ratio of the average spectral intensity of the CPC-tipped sensor and the plane-cut sensor at $618 \mathrm{~nm}$, which is where the peak of the fluorescence spectrum is located. The experimental results showed that the increment factor achieved with the CPC tip is 3.5. Numerical modeling with Zemax, using the ideal shape of the CPC with the same length and input and output diameters, predicts an increment factor of 3.96 .

The question is of course whether the Zemax predicted value of 3.96 is to be trusted, i.e., can it all be reached. After all, it uses several assumptions, such as one-scatter event per ray. ${ }^{6}$ To demonstrate the accuracy of the Zemax model, the actual, fabricated 
CPC shape is reconstructed in Zemax using freeform optics. The ideal and actual reconstructed CPC forms are compared in Fig. 4(a), which clearly demonstrates the accuracy of the fs-laser micromachining in generating the desired shape. Zemax calculations with the reconstructed actual fabricated shape then gave an increment factor of 3.62. The fact that this Zemax obtained values close to the experimentally found 3.5 allows us to conclude that the fs-laser micromachined CPC-tipped sensor has indeed an improved pickup efficiency close to the ideal value. It also allows us to trust that the predicted ideal value of 3.96 should be reachable with further optimization.

\section{Conclusion}

We have micromachined a CPC-shaped POF tip for enhancing the fluorescence pickup efficiency of fluorescence-based fiberoptical biosensors, such as the glucose sensor we have demonstrated here. The micromachined CPC tip showed an increment factor of 3.5 which is in close agreement with the Zemax predicted value of 3.62 obtained using the reconstructed manufactured CPC shape. This allowed us to conclude that the CPC shape obtained using the fs-laser micromachining is close to the ideal CPC shape and thus this method can be used to fabricate high-quality $\mathrm{CPC}$ tips in a controlled and precise manner. In terms of further improvement of the increment factor, it is important to note that Zemax predicts a value that is 0.12 higher than the measured value. In our similar comparison for CPC tips fabricated by heat-and-pull tapering using a soldering iron, ${ }^{6}$ we measured an increment factor of 2.1 where, using the reconstructed actual fabricated CPC shape, Zemax predicted a factor of around 2.3, so 0.2 higher. First, this shows that fs-laser micromachining is much more accurate that the heat-and-pull tapering. Second, it shows that it would be reasonable to assume that an increment factor of about 3.8 could be achieved with further optimization, since this is a reasonable 0.16 below the Zemax predicted ideal increment factor of 3.96 .

\section{Disclosures}

Authors declare that there is no conflict of interest regarding the publication of this paper.

\section{Acknowledgments}

The research leading to these results has received funding from the People Programme (Marie Curie Actions) of the European Union's Seventh Framework Programme FP7/2007-2013/under REA Grant Agreement No. 608382.

\section{References}

1. A. J. Müller et al., "First clinical evaluation of a new percutaneous optical fiber glucose sensor for continuous glucose monitoring in diabetes," J. Diabetes Sci. Technol. 7(1), 13-23 (2013).

2. J. C. Pickup et al., "Fluorescence intensity and lifetime-based glucose sensing using glucose/galactose-binding protein," J. Diabetes Sci. Technol. 7(1), 62-71 (2013).

3. R. Ballerstadt et al., "Fiber-coupled fluorescence affinity sensor for 3-day in vivo glucose sensing," J. Diabetes Sci. Technol. 1(3), 384-393 (2007).

4. A. Woehler, J. Wlodarczyk, and E. Neher, "Signal/noise analysis of FRET-based sensors," Biophys. J. 99(7), 2344-2354 (2010).

5. H. H. Gao et al., "Tapered fiber tips for fiber optic biosensors," Opt. Eng. 34(12), 3465-3470 (1995).

6. H. U. Hassan et al., "Polymer optical fiber compound parabolic concentrator tip for enhanced coupling efficiency for fluorescence based glucose sensors," Biomed. Opt. Express 6(12), 5008-5020 (2015).

7. W. T. Welford and R. Winston, The Optics of Nonimaging Concentrators, Academic, New York (1978).

8. A. Rabl, "Optical and thermal properties of compound parabolic concentrators," Solar Energy 18(6), 497-511 (1976).

9. L. C. Chen, A. L. Y. Low, and S. F. Chien, "Compound parabolic tapered fiber for fiber coupling with a highly divergent source," Appl. Opt. 43(32), 5923-5925 (2004).

10. R. R. Gattass and E. Mazur, "Femtosecond laser micromachining in transparent materials," Nat. Photonics 2, 219-225 (2008).

11. M. Dubov, I. Khrushchev, and I. Bennion, "Direct writing of fibre Bragg gratings by femtosecond laser," Electron. Lett. 40(19), 1-2 (2004).

12. K. M. Davis et al., "Writing waveguides in glass with a femtosecond laser," Opt. Lett. 21(21), 1729-1731 (1996).

13. A. M. Streltsov and N. F. Borrelli, "Study of femtosecond-laserwritten waveguides in glasses," J. Opt. Soc. Am. B 19(10), 2496-2504 (2002).

14. K. Minoshima et al., "Photonic device fabrication in glass by use of nonlinear materials processing with a femtosecond laser oscillator," Opt. Lett. 26(19), 1516-1518 (2001).

15. K. Minoshima et al., "Fabrication of coupled mode photonic devices in glass by nonlinear femtosecond laser materials processing," Opt. Express 10(15), 645-652 (2002).

16. Y. Sikorski et al., "Optical waveguide amplifier in Nd-doped glass written with near-IR femtosecond laser pulses," Electron. Lett. 36(3), 226-227 (2000).

17. L. Ding et al., "Optimization of femtosecond laser micromachining in hydrogel polymers," J. Opt. Soc. Am. B. 26(9), 1679-1687 (2009).

18. C. R. Mendonca et al., "Femtosecond laser micromachining in the conjugated polymer MEH-PPV," Appl. Surf. Sci. 254(4), 1135-1139 (2007).

19. P. J. Scully, D. Jones, and D. A. Jaroszynski, "Femtosecond laser irradiation of polymethylmethacrylate for refractive index gratings," J. Opt. A 5(4), S92-S96 (2003).

20. A. Lacraz et al., "Femtosecond laser inscribed Bragg gratings in low loss CYTOP polymer optical fiber," IEEE Photonics Technol. Lett. 27(7), 693-696 (2015).

21. B. Cai et al., "A three-dimensional polymeric optical circuit fabrication using a femtosecond laser-assisted self-written waveguide technique," Appl. Phys. Lett. 92, 253302 (2005).

22. R. Vázquez et al., "Fabrication of binary Fresnel lenses in PMMA by femtosecond laser surface ablation," Opt. Express 19(12), 11597-11604 (2011).

23. A. A. Said et al., "Carving fiber-top cantilevers with femtosecond laser micromachining," J. Micromech. Microeng. 18(3), 035005 (2008).

24. L. Yuan et al., "Fiber inline Michelson interferometer fabricated by a femtosecond laser," Opt. Lett. 37(21), 4489-4491 (2012).

25. A. Ioannou et al., "Beam-shaping via femtosecond laser-modified optical fibre end faces," Proc. SPIE 9886, 98860J (2016).

26. A. Bottino et al., "Solubility parameters of poly(vinylidene fluoride)," J. Polym. Sci. B Polym. Phys. 26(4), 785-794 (1998).

27. R. M. Clegg, "Fluorescence resonance energy transfer," Curr. Opin. Biotechnol. 6(1), 103-110 (1995).

28. E. Baur, K. Ruhrberg, and W. Woishnis, Chemical Resistance of Engineering Thermoplatics, 1st ed., William Andrew, UK (2016).

Hafeez UI Hassan received his BS degree in engineering sciences from Ghulam Ishaq Khan Institute of Engineering Sciences \& Technology, Pakistan, in 2008, and his MSc degree in photonics from Friedrich Schiller University, Jena, Germany, in 2011. He received his $\mathrm{PhD}$ in photonic engineering from Technical University of Denmark in 2017. His research interests are optical fiber and fiber sensors.

Amédée Lacraz holds a MSc in physics at École Supérieure de Physique et de Chimie Industrielles in Paris and a MSc in optics and photonics at Institut d'Optique in Palaiseau, where he was trained in theoretical physics as well as optical engineering. He received his PhD from Cyprus University of Technology in 2017. His research interests include femtosecond laser based inscription on polymer optical fiber. 
Kyriacos Kalli is an associate professor in the Department of Electrical Engineering, Computer Engineering, and Informatics at Cyprus University of Technology. He received his BSc (Hons) in theoretical physics in 1988, and his $\mathrm{PhD}$ in physics from the University of Kent, UK, in 1992, where he studied linear and nonlinear phenomena in optical fibres. He is a member of the IOP, and IEEE and a senior member of the OSA. His research interests are in Bragg grating, fibre sensors, femtosecond laser microfabrication in glass and polymers, microfluidics, and plasmonics.
Ole Bang is a professor at the Technical University of Denmark and fellow of OSA. He heads the Fiber Sensors \& Supercontinuum Group, which develops photonic crystal fibers for sensing and supercontinuum lasers for applications in imaging and spectroscopy. He has published 180 journal papers with 6040 ISI citations and ISI h-index 44 . He holds several patents within supercontinuum lasers and polymer optical fiber sensors and is cofounder of SHUTE Sensing Technologies. 